\title{
THE RIGID FORM OF HUNTINGTON'S DISEASE
}

\author{
BY
}

\section{A. M. G. CAMPBELL, BERYL CORNER, R. M. NORMAN, and H. URICH \\ From the Department of Neurosurgery and Child Health, Bristol Royal Hospital, and the Burden Neuropathological Laboratory, Frenchay Hospital, Bristol}

Although the majority of cases of hereditary chorea correspond accurately to the classical pattern described by Huntington (1872), a number of atypical forms have been recorded in children and adults which are characterized by rigidity rather than by hyperkinesia. Most of these have been reported in the continental literature and we thought it was of interest to draw attention to two atypical juvenile cases occurring in an English family.

From Reisner's (1944) review of these juvenile cases the fact emerges that although the majority present with typical choreiform movements, two atypical variants also occur: one in which the clinical picture is that of progressive extrapyramidal rigidity without involuntary movements, the other in which the disease starts as a hyperkinetic syndrome and gradually changes into a hypokinetic one with progressive rigidity. These two syndromes are commoner in the juvenile group, though occasional similar cases have been recorded in adults, in some instances affecting entire families, like that of Schob (1927), in which rigidity followed a preliminary stage of chorea, and that of Hallervorden (1957), in which rigidity was the predominant symptom throughout the course of the illness. Finally, there is the unusual case briefly recorded by Westphal (1905). This patient came from a family with seven other members affected by Huntington's chorea. Choreic movements in this patient developed late in the course of the disease, mental abnormality having been observed for 12 years, and Parkinsonian rigidity and intention tremor having been present for seven years before the onset of typical hyperkinesia. No post-mortem findings were described.

Clinical accounts of these atypical variants, both juvenile and adult, are fairly numerous (see Hallervorden, 1957) but detailed pathological studies are rare. The classical description of juvenile cases which never exhibited choreiform movements is that of Spielmeyer $(1920,1926)$ who described two cases recorded clinically (as instances of Wilson's disease) by Kraepelin (1921) and shown by the genetic study of Entres (1925) to belong to a typical Huntington family. More recent contributions are those of Rotter (1932), Hempel (1938), and Lindenberg (1960). Bielschowsky (1922) gave a detailed account of the pathological findings in a patient who was choreic at the age of 6 years and from the age of $\mathbf{9}$ gradually developed Parkinsonian rigidity. Our own juvenile case is remarkable in

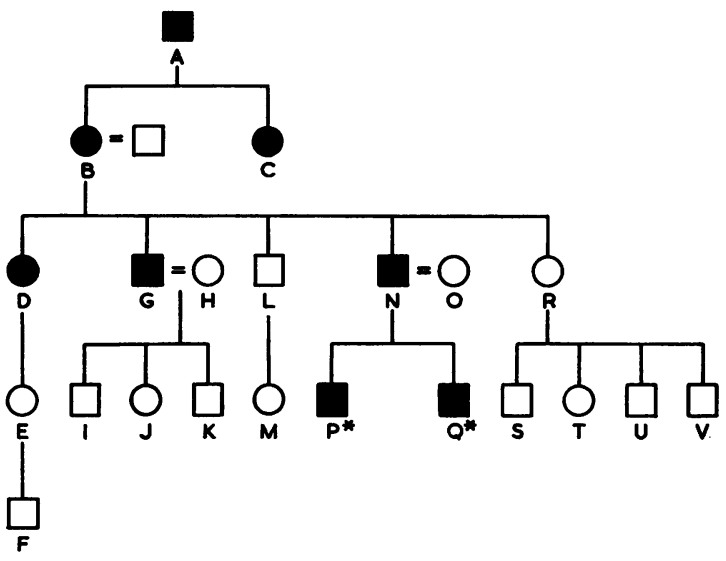

Family tree made in December, 1956.

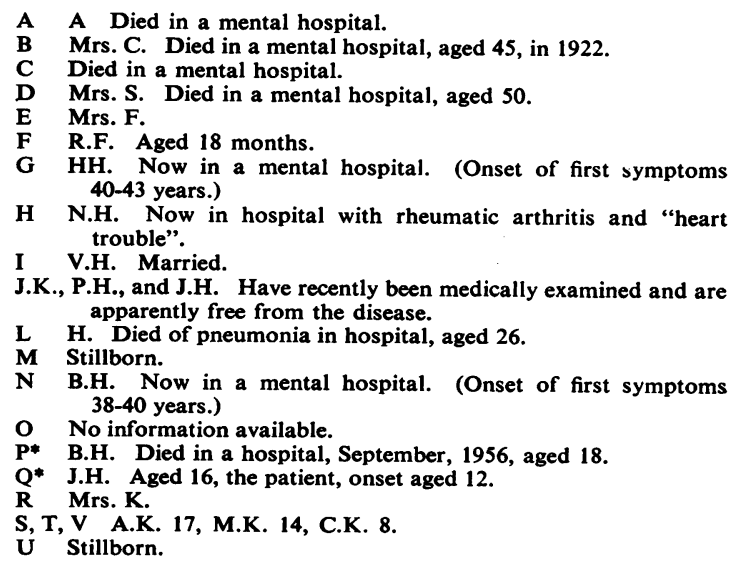


that mild choreic movements became established after the Parkinsonism had been present for about seven years. This sequence of events thus resembles that in Westphal's case.

The family we have studied lived in Bristol but had relatives who were traced back to Scottish ancestry and Cornwall. The older members of the family do not concern us in this paper, but two of them have been seen by one of us (A.M.G.C.) numbered $G$. and $N$. in the family tree. These were classical cases of Huntington's chorea presenting with mental symptoms and choreiform movements at the age of about 40 . Both had been apparently normal during the earlier years of their life and able to hold reasonable jobs until the onset of the disease. Both are now confined to a mental hospital. Case N. had two sons who form the basis of this particular paper. The following is the case history of the first boy who was under the care of one of us (B.C.).

\section{Case Reports}

B.H., the first child in this family, was seen at the age of 10 years 2 months on account of severe attacks of asthma which began during his third year. A very undersized child with the weight and body measurements of a boy of only 7 years, he had severe pulmonary emphysema, consolidation of the left lower lobe, and antral sinusitis, for which he was admitted to hospital for investigation.

It was then observed that he was a very shy, immature boy, who made no attempt to play or converse with other children in the ward. The striking features were his expressionless face and generalized rigidity. No involuntary movements were observed. Hand movements were so poorly coordinated that he was unable to wash or dress himself, and he fed himself with obvious difficulty and very messily. His voice was low pitched and articulation poor. On questioning, his mother admitted that she had noticed increasing stiffness of his legs and difficulty in walking for about a year. A diagnosis of Parkinsonism, possibly post-encephalitic, was made.

During the next two years his physical condition steadily deteriorated. Generalized rigidity and shuffling gait increased, a fine tremor developed, and he became almost inarticulate. All deep reflexes and abdominal reflexes were brisk, and plantar responses flexor. No sensory changes were elicited. Treatment with artane was tried without effect.

No abnormality was seen in the cerebrospinal fluid.

Air encephalography showed in the lateral ventricles slight dilatation, in the left greater than in the right.

No alpha activity was detected in the E.E.G., but diffuse delta and theta waves from all areas.

The Wassermann and Kahn reactions were negative. The blood group was $\mathrm{A} \mathrm{Rh}$ positive, the blood count normal, and plasma proteins normal.

Thymol turbidity was 4 units, thymol flocculation partial, colloidal gold flocculation 2 units, and plasma phosphatase 14 units.

Urinary copper and amino-acid excretion was within: normal limits.

His I. Q. (Terman Merrill) was 76, and arithmetiç ability was grossly retarded.

Two years and nine months after he was first seen his paternal aunt died in hospital having been diagnosed aş a possible case of Huntington's chorea, and his fathee. was in hospital with the same condition. Shortly afte this, at 13 years of age, the boy had two severe major. fits, and three months later typical choreiform move $\Rightarrow$ ments developed in the left hand. He also complained of? persistent itching of the dorsum of both feet. During the next two months the fine tremor greatly increased and there were also continuous choreiform movements ins both legs and arms. He was still attending a day schood for physically handicapped children but with frequent absences owing to asthma.

At the age of 15 years he was again admitted to hcspita as the fits had now become very severe and occurredalmost daily. Mentally he was cooperative and slightl $\overrightarrow{\mathbf{w}_{\mathbf{w}}}$ euphoric, but responsive within the limits of his motor disability. He was still able to read, and was interesteç in his surroundings.

During the subsequent months there was very rapid deterioration in his physical and mental state so that shortly before his sixteenth birthday he was admitfed to a hospital for the chronic sick, bedridden, advanced pseudo-bulbar palsy and severe mentå deterioration. An E.E.G. at this time showed gene苗ized abnormality as before. He died at the age of years 11 months.

The second boy, J.H., case Q, closely resembled क्षi brother in his clinical features. At the age of 14 the mother noticed that the boy began to get slow clumsy in his movements. His mother also noticed thatho had a tendency to fall; the mother was, of course, aware at this time of the family history and of the brother' illness, and she was on the look-out for the developmen of symptoms in this particular boy. He apparently ha quite a good school record, was not brilliant but now noticeably backward. He left school at the age of 15 and worked in a mattress factory.

Recently he has found that he is unable to carry the heavy loads he could manage formerly. On examination it was noticed that he had an immobile facial expressio and that he had marked rigidity resembling the rigidit\$. of Parkinsonism. He moved with difficulty and slowly his speech was slightly slurred, and coordination of movements was impaired. He also showed generalize $\bar{\Phi}$ extrapyramidal cogwheel rigidity throughout all fou limbs. His gait was stiff and there was a marked tendency not to swing his arms, particularly the left one.

This boy has gradually deteriorated over the thre years he has been under observation, although it is obvious that he is not unintelligent, but unfortunately he has an insight into the possible future development his disease in a similar way to that of his brother. Durin the last year of observation a certain number of inf voluntary movements have become obvious in the left hand, but rigidity still dominates the picture and when he 
was shown at a clinical meeting about 18 months ago, without the family history being divulged, the majority of clinicians decided he must be a case of postencephalitic Parkinsonism.

The interest, therefore, of these two cases is, first, the precocious development of the disease in these two boys compared with the course in their ancestors who developed the disease at a much later age, and secondly, the development of rigidity as a predominant feature rather than involuntary movements or mental changes.

\section{Examination of the Central Nervous System in Case 1}

Macroscopic.-The fixed brain weighed 1,172 g., the cerebellum and brain-stem accounting for $178 \mathrm{~g}$. The convolutional pattern was normal but the sulci were widened over the convexity of the cerebral hemispheres, particularly so in the central region and in the occipital lobes. On coronal section the lateral ventricles were markedly dilated in symmetrical fashion and severe atrophy was seen in the caudate nuclei and putamina (Fig. 1). The globus pallidus of each side was also shrunken but relatively less so than the striata. The blood vessels and meninges were unremarkable.

Microscopic.-Large representative blocks of the cerebral hemispheres, basal ganglia, cerebellum, and brain-stem were embedded in celloidin and sections stained for nerve cells and myelin. Frozen sections were stained for myelin, nerve cells, lipid, and fibrous neuroglia. On the right side the basal ganglia were examined at millimetre levels from the mammillary bodies to the terminal part of the globus pallidus. On the left side, hemisphere sections were similarly examined from the middle of the nucleus subthalamicus to the posterior termination of the external geniculate body.

Cerebral Cortex.-Both postcentral gyri were severely shrunken and showed a substantial and diffuse loss of nerve cells mainly in the third and fifth layers (Fig. 2). In the precentral gyrus similar neuronal losses were more patchily distributed, the giant cells of Betz being well preserved but crowded together. A generalized reduction in the depth of the cortex was commonly seen (Figs. 3a, 3b). In places the two lowest layers of the motor cortex were the most affected. Nerve cells were widely destroyed in both occipital lobes, mainly in the smaller cells of the third layer but often diffusely, as in the parastriate cortex (Fig. 4). In the visual cortex changes were less intense and the deeper part of the internal granular layer was usually the best preserved in the more affected gyri. In much of the prefrontal, parietal, and temporal areas the architecture of the cortex was well preserved though patchy areas of nerve cell loss were common and many of the nerve cell bodies appeared slightly shrunken. A selective degeneration of extensive parts of the internal granular layer was frequently seen in the temporal isocortex. The hippocampal formations were intact. No lipid granules or excess of lipochrome were seen in the nerve cells. The supraradial myelinated fibres were scanty in the better preserved gyri and in the atrophied regions the radial fibres were also feebly represented. The destruction of nerve cells was not associated with fibrillary gliosis except in the molecular layer and to a less extent in the outer part of the grey matter. The centrum ovale and the medullary cores of many gyri were markedly reduced in volume. In general, no demyelination was demonstrated in frozen sections, though in celloidin-embedded material the staining of myelin was often not so intense as in normal brains. In the atrophied occipital and central regions the white matter showed a mild, diffuse, fibrillary gliosis and a few fat granule cells were occasionally seen around vessels.

Basal Ganglia.-Both striate bodies were grossly atrophied, the caudate nuclei being represented by an elongated thin strip of tissue and the putamina severely shrunken to about one-third of the normal size (Fig. 1). The small nerve cells had virtually disappeared while many of the larger ones had survived and were crowded together (Fig. 5). There was a marked increase of glial nuclei throughout the whole striatum but a fibrillary gliosis was demonstrated only in the putamen (Fig. 6). The strio-pallidal fibre bundles were thin, rather poorly myelinated, and showed an apparent increase of oligodendroglia.

The globus pallidus was also shrunken, its area in cross section being about half that of normal. In the anterior part of the nucleus the nerve cells appeared healthy though more closely packed together than in the normal adult. There was a marked increase in oligodendroglial nuclei (Fig. 7a). Myelination was not reduced and no pallor or gliosis was seen in the position of the "strio-nigral tract". More posteriorly the pallidal fibres stained poorly in myelin preparations and areas could be found in the external segment in which the nerve cells were less numerous and individually very small and poor in Nissl substance (Fig. 7b). Fibrillary gliosis was marked in the posterior part of the nucleus. The divisions of the ansa lenticularis were identified and appeared normal except for slightly reduced intensity of myelin staining. 


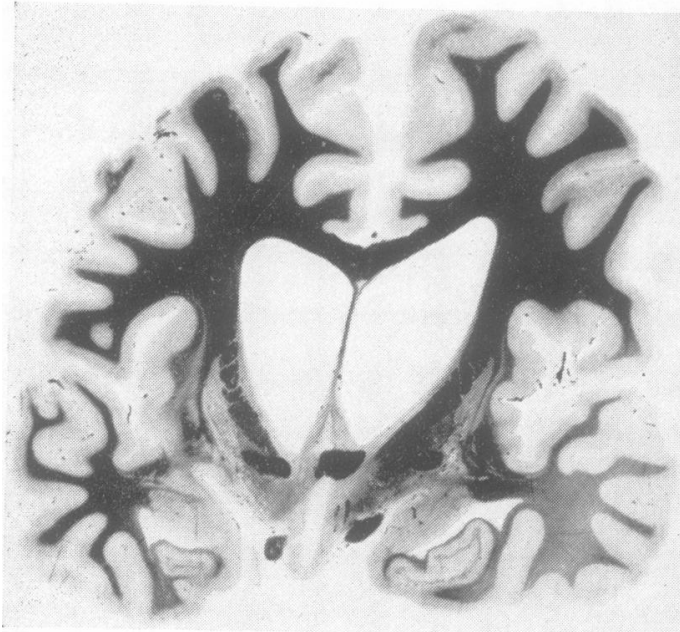

FIG. 1.-Coronal section through the frontal lobes showing marked atrophy of the centrum ovale, caudate nuclei, and putamina. Heidenhain $\times 0.8$.

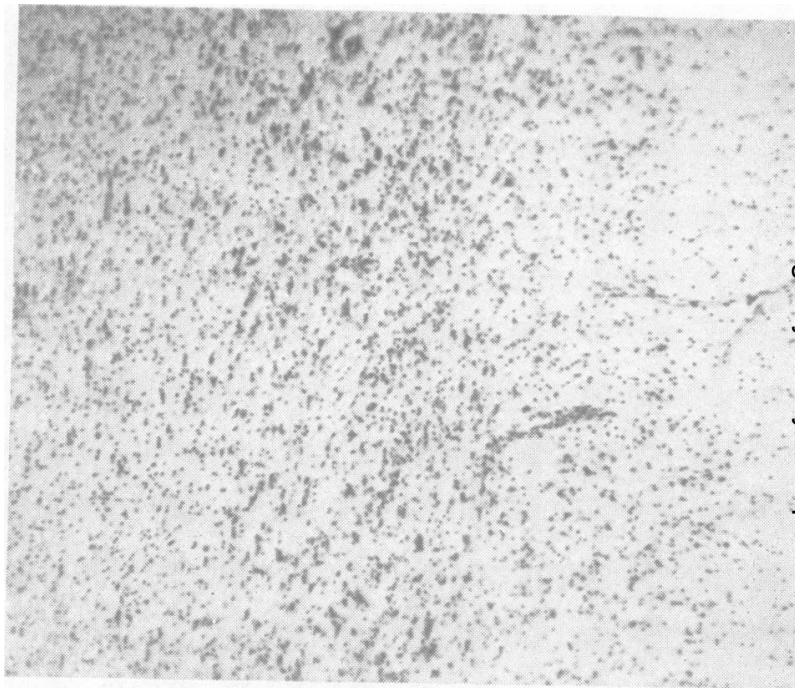
Fig. 2.-Postcentral gyrus showing diffuse degeneration of nerve cells. $\overrightarrow{C_{a}}$ arb
azure $\times 80$.

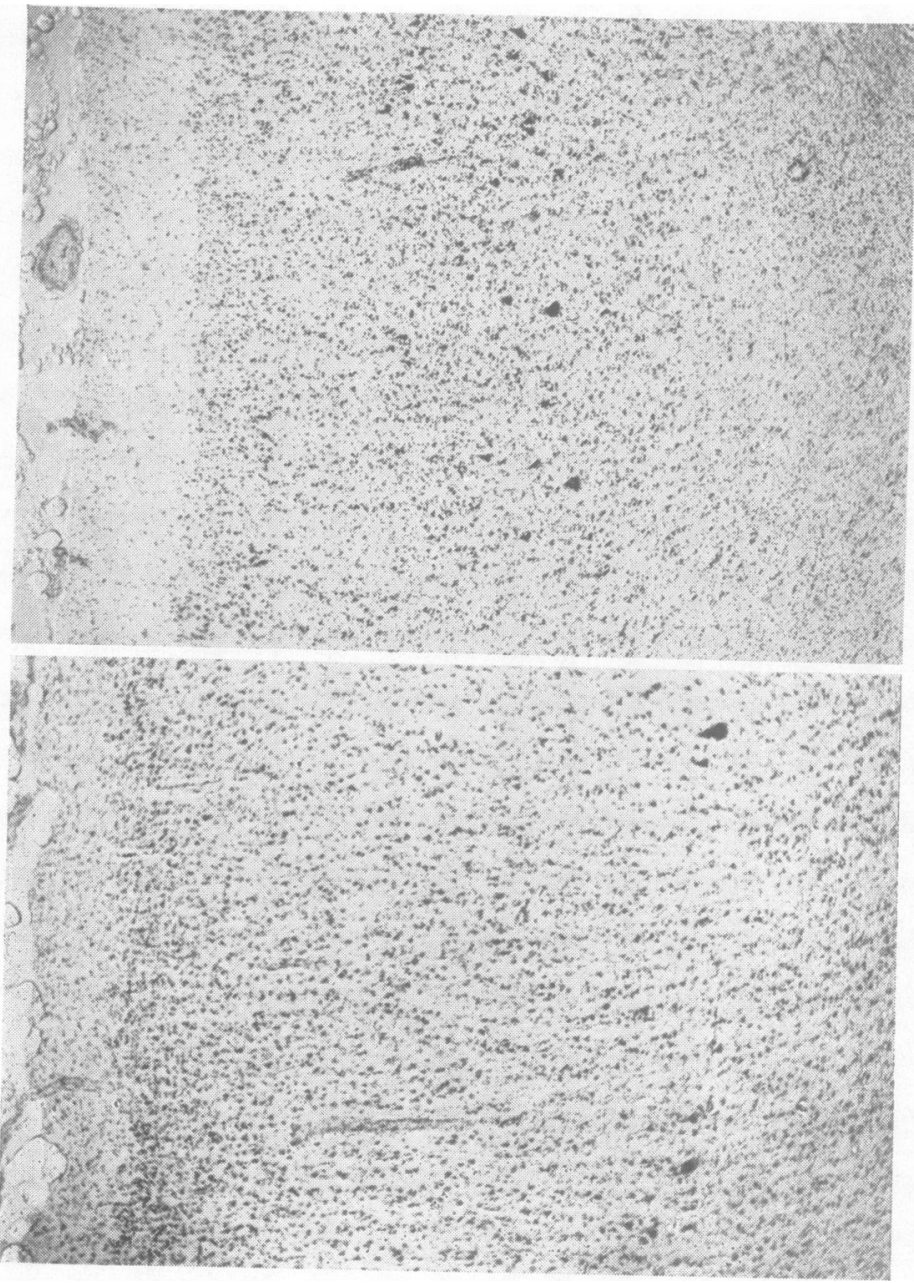

Fic. 3a.- Precentral gyrus showing generalized reduction in the depth of the grey matter. 3b. - Normal control. Carbol azure $\times 40$. of $\mathrm{L} 3$ are relatively preserved. Carbol azure 75 . 


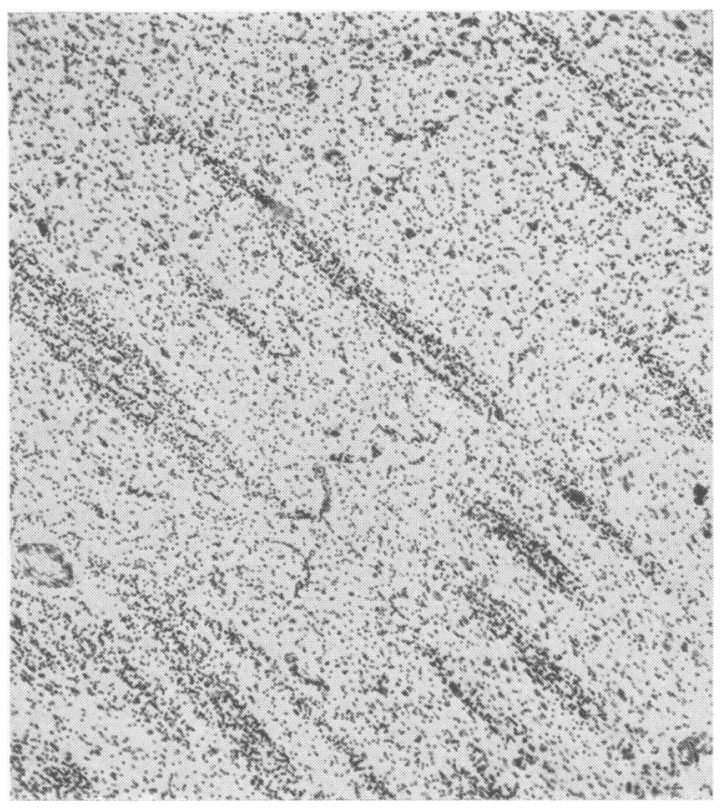

FIG. 5.-Putamen. The small nerve cells are almost completely lost. The astrocytic nuclei are increased in number. The striopallidal fibre bundles are crowded together and show an excess of oligodendroglial nuclei. Carbol azure $\times 60$.

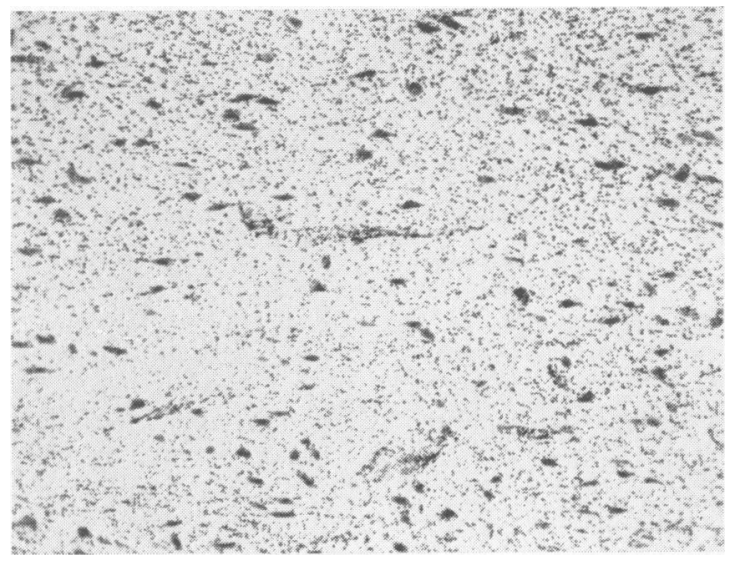

FrG. 7a.-Globus pallidus, anterior level. The nerve cells are normal in appearance but are unduly crowded together. Carbol azure $\times 60$.

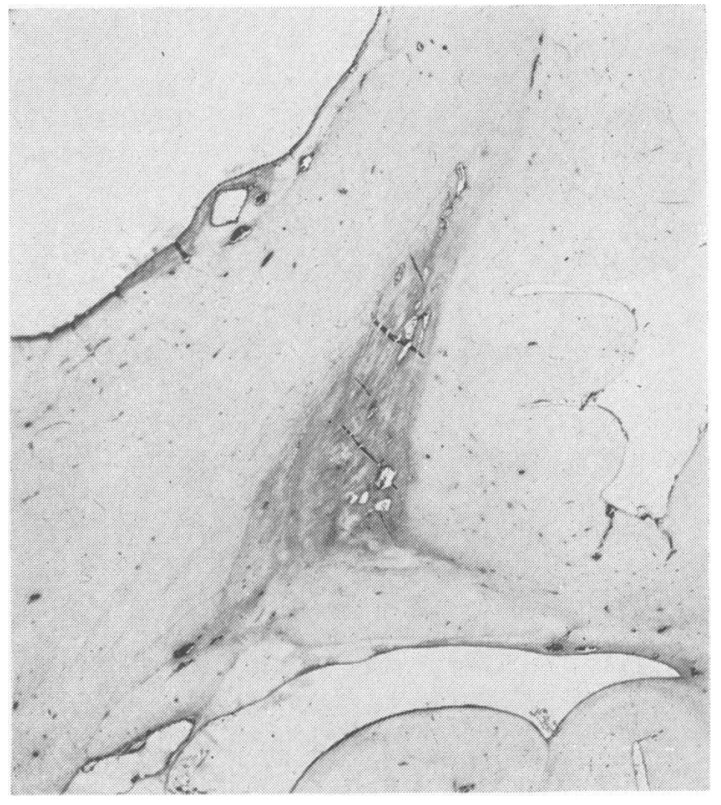

Fig. 6.-Fibrillary gliosis in the putamen and posterior part of the globus pallidus. Holzer $\times 3$.

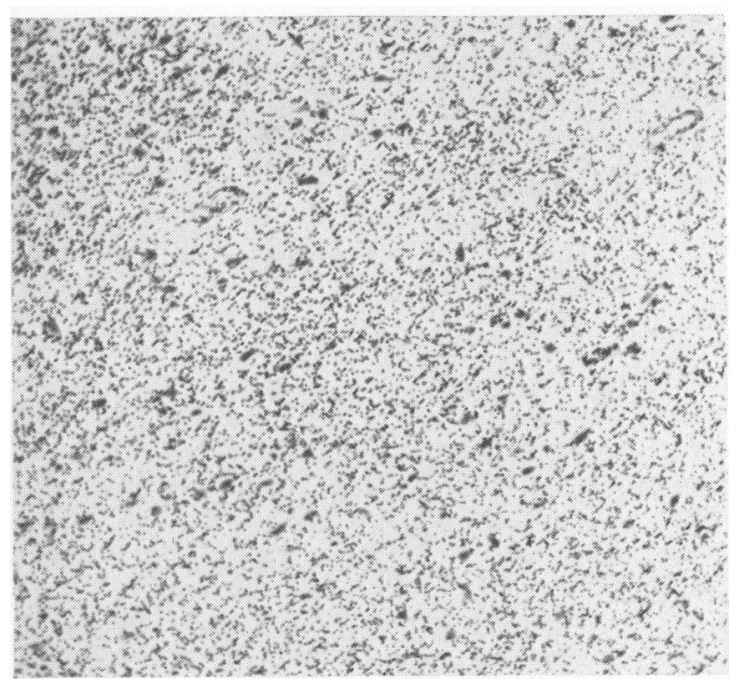

FIG. 7b.-Globus pallidus, posterior level. There is a reduction in the numbers of nerve cells, many of which are shrunken. Carbol azure $\times 60$. 
The claustrum and amygdaloid nucleus were intact.

The thalamus appeared somewhat flattened laterally in coronal sections but no significant loss of nerve cells was found in the various nuclei. Detailed examination of the corpora Luysii revealed no lesion.

The lateral lobes of the cerebellum, vermis, and dentate nuclei were normal.

Brain-stem.-Although no loss of nerve cells could be demonstrated in the substantia nigra the neurones generally appeared smaller and contained less melanin pigment than those of the normal adult. The cell groups as a whole had shrunk a little and the cytons appeared rather crowded together. The red zone was markedly narrow and in places contained an excess of glial nuclei. The red nucleus appeared to be normal. The cerebellar peduncles and other tracts and nuclei were unaffected. The pons appeared normal except for marked gliosis and a slight loss of nerve cells in the superior olives.

No pathological changes were seen in the medulla. In the spinal cord there was a distinct pallor of myelin staining in the peripheral zone of the anterior cord lateral columns throughout the cord. The nerve cells were unremarkable.

\section{Discussion}

Attempts to distinguish pathologically between the choreic and akinetic forms of Huntington's disease have not revealed clear-cut differences despite the striking clinical contrast. Bielschowsky's (1922) conclusion that the rigidity of his patients could be ascribed to the severity of the pallidal lesions was contraverted by Spielmeyer (1926) who pointed out that the pallidal changes in his own rigid cases were less marked than those often found in purely choreic patients. In certain instances pallidal lesions have been found in combination with degeneration of the large striatal nerve cells (Jakob, 1923) but the latter feature is not usually seen in the akinetic type of the disease and cannot be regarded as a significant factor in the production of rigidity.

In the light of recent neurosurgical experience and from the results of animal experiments this simple correlation of pallidal lesions with rigidity must be dismissed as obsolete. Yet it is well to bear in mind that the beneficial effects of pallidectomy on hyperk:nesia depend upon relatively minor pallidal damage, only about $10 \%$ of the whole nucleus being involved (Carpenter, Whittier, and Mettler, 1950). One cannot safely assume that very extensive pallidal destruction is similarly innocuous, otherwise the crippling disabilities of kernicterus and pro- $\frac{\text { O }}{Z}$ gressive pallidal degeneration (van Bogaert, 1946)థ would be inexplicable. In Huntington's disease, however, this consideration does not apply, for the pallidum may be relatively spared both in theô choreic and akinetic forms.

Later authors who have compared the two variants from the pathological point of view have been impressed by the more massive character of the lesions in the rigid cases, though they have found that the same structures may be involved in $\overrightarrow{\vec{F}}$ both forms. Not only are the striatal lesions very severe and diffuse but there is usually a marked ${ }^{\circ}$ generalized cerebral atrophy with considerable ven- $\overline{\bar{\omega}}$ tricular dilatation and brain weights below $1,000 \mathrm{~g}$. (Meyjes, 1931; Rotter, 1932; Hempel, 1938). There is thus a substantial, though ill-defined, $\infty$ cortical component in the syndrome. In our case $\vec{O}$ the brain weighed $1,172 \mathrm{~g}$. so that the atrophy of the $\overrightarrow{\vec{H}}$ centrum ovale was evidently less than may occur in $\vec{\omega}$ the akinetic cases. On the other hand, the severity of the nerve cell loss in the caudate nucleus and putamen was fully equivalent to what has been i described in all the rigid cases. The solution of the problem awaits the quantitative assessment of the damage suffered by the various nuclei concernee in the regulation of involuntary movement and muscle tone, and this is a task of great difficulty. A円 estimate can, however, be made on the basis \& 8 serial sections and certain findings in the preserf cases, particularly the integrity of the corpus Luys and substantia nigra, appear worthy of commeng. when set against the background of Purdon Martin $\$$ $(1959,1960)$ recent analysis of basal ganglia function.

In the first place the Parkinsonian rigidity in our case was not associated with a loss of nerve cells in the compact zone of the substantia nigra. This is also true of other juvenile rigid types of Huntington's disease described in the literature. A rare striopallidal-nigral syndrome, also characterized by rigidity, has been reported by Vander Eecken, Adams, and van Bogaert (1960) but this appears to be a distinct pathological entity and its genetic relationship to Huntington's chorea not yet established. If, then, the rigidity in our case was due to the overaction of the pallidum, that is to say, by the operation of the "supplementary tone-producing mechanism" suggested by Martin, the release of excessive pallidal activity must have been due to lesions in a centre or centres other than the substantia nigra. Although the globus pallidus was shrunken the nerve cells were largely preserved, especially in the medial segment, and the divisions of the ansa were intact. There were also no detectable lesions in the thalamus. It would thus appear that an essential

\section{.}


mechanism for the production of rigidity (or hyperkinesia) had been relatively preserved. It may be suggested, though it cannot be proved, that this release of pallidal activity may have been conditioned by the severe degeneration of the putamen. As Martin (1959) has pointed out, "not all extrapyramidal rigidity is associated with significant disease of the substantia nigra. Wilson's disease provides the best example of the contrary and ... in this condition the striatum suffers severely". This statement is in harmony with the views expressed by Denny-Brown (1960) on the causation of rigidity in Huntington's disease in his recent Croonian lectures. In a personal communication he writes: "The essential point is that rigidity ensues when the inner part of the putamen becomes seriously damaged". A corollary to this hypothesis would be that early and severe degeneration of the putamen may be responsible for the purely akinetic varient of the disease. It may be of significance that in our case a dense fibrillary gliosis was present in the putaminal part of the striatum and not in the caudate nucleus.

The second point of interest raised by this case concerns the appearance of mild choreic movements late in the course of the patient's illness. Evidently the structures responsible for this manifestation had not been destroyed or their action permanently suppressed by the lesions causing the initial state of rigidity. Important findings were the complete escape of the bodies of Luys and of the dentatorubral system. There remain the lesions of the caudate nuclei and the cerebral cortex as sources of that functional upset in the balance of power which seem to underlie choretic movements. The experiments of Kennard and Fulton (1942) have shown that in the chimpanzee true unilateral chorea followed combined extirpation of cortical area 6 and the caudate nucleus of the opposite side. The findings in our case were not so clear cut, for although there was no doubt as to the severity of the caudate nuclear degeneration, the prefrontal region merely shared the generalized cortical atrophy and was not preferentially involved. It seems advisable, however, to admit the possibility of a cortical component in the hyperkinesia of Huntington's disease, since the evidence favouring purely striatal lesions as a cause of chorea is controversial (Meyers, 1942).

We therefore suggest that the choreic and akinetic types of Huntington's disease owe their contrasting clinical features to the relative severity of the caudate-cortical degeneration on the one hand and of the putaminal degeneration on the other. The temporal sequence of these lesions must also have a decisive influence on the clinical picture.

\section{Summary}

Details have been given of an English family in which classical Huntington's chorea has been traced for three generations. In the fourth generation two brothers suffered from the juvenile, rigid form of the disease. In the elder a Parkinsonian rigidity began at about the age of 10 years. At the age of 13 this patient began to have major epileptic fits and choreiform movements in the limbs appeared for the first time. He died bedridden and with advanced pseudo-bulbar palsy at the age of 17 years. Neuropathological examination showed widespread cerebral cortical atrophy with maximal involvement of the postcentral gyri and the occipital lobes. The centrum ovale was atrophied and the lateral ventricles considerably enlarged. Severe symmetrical atrophy affected the caudate nuclei and putamina from which the small nerve cells had largely disappeared. The globus pallidus was shrunken but there was little loss of nerve cells except in the external segment. The compact zone of the substantia nigra was normal except for slight general shrinkage. No lesions were found in the corposa Luysii or dentato-rubral system. It is suggested that the akinetic form of Huntington's disease is primarily characterized by the severity of the putaminal lesions, while the choreic manifestations of this disorder are due to involvement of the caudate nuclei, perhaps in association with their cerebral cortical connexions.

We are much indebted to Dr. Purdon Martin for his advice on many problems arising during the preparation of this paper. The neuropathological part of this work was assisted by the Nuffield Foundation.

\section{REFERENCES}

Bielschowsky, M. (1922). J. Psychol. Neurol. (Lpz.), 27, 233.

Bogaert, L. van (1946). J. Neurol. Neurosurg. Psychiat., 9, 125.

Carpenter, M. B., Whittier, J. R., and Mettler, F. A. (1950). J. comp. Neurol., 92, 293.

Denny Brown, D. (1960). Lancet, 2, 1099 and 1155.

Entres, J. L. (1925). Z. ges. Neurol., 98, 497.

Hallervorden, J. (1957). In Henke-Lubarsch Handbuch der speziellen, pathologischen Anatomie und Histologie, ed. O. Lubarsch, pathologischen Anatomie und Histologie, ed. O. Lubarsch,
F. Henke and R. Rössle, Band 13, teil 1, bandteil A, p. 811 .

Springer, Berlin.
Hempel, H. C. (1938). Z. ges. Neurol., 160, 563.

Huntington, G. (1872). Med. surg. Reporter, $26,317$.

Kennard, M. A., and Fulton, J. F. (1942). Proc. Ass. Res. nerv. ment. Dis. (Baltimore), 21, 228.

Jakob, A. (1923). Die extrapyramidalen Erkrankungen. Springer Berlin. Kraepelin, E. (1921). Einführung in die psychiatrische Klinik, 4th ed., Vol. 3, p. 156. Barth, Leipzig.

Lindenberg, R. (1960). J. Neuropath., 19, 160

Lindenberg, R. (1960). J. Neuropath.
Martin, J. P. (1959). Lancet, 1, 999.

Martin, J. P. (1959). Lance

Meyers, R. (1942). Proc. Ass. Res. nerv. ment. Dis. (Baltimore), $21,602$.

Meyjes, F. E. P. (1931). Z. ges. Neurol., 133, 1.

Reisner, H. (1944). Nervenarzt., 17, 86.

Rotter, R. (1932). Z. ges. Neurol., 138, 376.

Schob, F. (1927). Mschr. Psychiat. Neurol., 65, 285.

Spielmeyer, W. (1920). Z. ges. Neurol., 57, 312.

(1926). Ibid., 101, 701 .

Vander Eecken, H. V., Adams, R. D., and Bogaert, L. van (1960). J. Neuropath. i9, 159.

Westphal, A. (1905).' Zbl. Nervenheilk., 28, (n.s. 16), 674. 